Z. Klin. Chem. Klin. Biochem.

13. Jg. 1975 , S. 499-505

\title{
Einfluß verschiedener Methoden zur Blutgewinnung auf Enzym-Aktivitäten im Serum kleiner Laboratoriumstiere $^{1,2}$ )
}

\author{
Von R. Friedel, I. Trautschold, K. Gärtner, M. Helle-Feldmann und D. Gaudssuhn \\ Institut für Klinische Biochemie und Zentrales Tierlaboratorium der Medizinischen Hochschule Hannover
}

(Eingegangen am 7. April/10. Juni 1975)

Zusammenfassung: Die Höhe der Aktivitäten von Zellenzymen im Serum von Ratte und Maus wurde auf ihre Abhängigkeit von der Technik der Blutentnahme untersucht. Bei der Ratte wurde Blut entnommen durch Punktion des retroorbitalen Venenplexus, über einen in eine Vena jugularis eingeführten Katheter, durch Herzpunktion und durch Incision der abdominalen Aorta; bei der Maus über einen Venenkatheter und durch Aortenincision. Im Serum wurden die Aktivitäten folgender Zellenzyme bestimmt: Sorbitdehydrogenase, Lactatdehydrogenase, Malatdehydrogenase, Glutamatdehydrogenase, Aspartataminotransferase, Alaninaminotransferase, Pyruvatkinase, Creatinkinase, Myokinase, Alkalische Phosphatase und Leucinaminopeptidase. Bei der Ratte wurden zusätzlich die Konzentrationen von Protein, Harnstoff und anorganischem Phosphat sowie die Hämatokritwerte bestimmt. Versuchsbedingte Änderungen der Größe des intravasalen Flüssigkeitsraumes wurden durch Untersuchung der Verteilung von intravenös injiziertem ${ }^{125} \mathrm{~J}$-markiertem Albumin erfaßt.

Bei der Ratte wurden sowohl nach Retroorbitalpunktion als auch nach Aortenincision im Serum höhere Aktivitäten für Lactatdehydrogenase, Malatdehydrogenase, Aspartataminotransferase, Pyruvatkinase, Creatinkinase und Myokinase gefunden als bei Herzpunktion oder Venenkatheterisierung. Alkalische Phosphatase und Alaninaminotransferase wurden nur nach Retroorbitalpunktion mit leicht zu hohen Aktivitäten im Serum bestimmt. Nach Herzpunktion wurden lediglich für die Creatinkinase zu hohe Werte gefunden. Zu hohe Proteinkonzentrationen und Hämatokritwerte nach Retroorbitalpunktion sowie zu niedrige Meßwerte für beide Parameter nach Aortenincision sind auf eine durch den Versuch bedingte Einengung bzw. Vergrößerung des relativen Plasmaraumes zurückzuführen. Bei der Maus wurden nach Aortenincision Lactatdehydrogenase, Malatdehydrogenase, Aspartataminotransferase, Creatinkinase und Myokinase mit zu hohen Aktivitäten im Serum gefunden.

Die Ursache für die Unterschiede in den Enzymaktivitäten wird weniger in Einflüssen durch Hämolyse oder Blutgerinnung als vielmehr in einem durch den. Eingriff verursachten Übertritt von Enzymaktivität vom intrazellulären in den extrazellulären Raum gesehen. Für Untersuchungen von Enzymaktivitäten im Serum von Ratte und Maus ist die Blutentnahme über einen Venenkatheter die Methode der Wahl. Für chronische Versuche wird die Herzpunktion empfohlen.

\section{Effects of blood sampling on enzyme activities in the serum of small laboratory animals}

Summary: Because of the difficulties in drawing blood for clinical chemistry in small laboratory animals there exist many methods for sampling blood and the preparation of serum, none of which is generally accepted or well standardised. It was the aim of this study to investigate the effects of sampling techniques on normal values of enzyme activities in the serum of rat and mouse.

The activities of the following enzymes were determined: sorbitol dehydrogenase, lactate dehydrogenase, malate dehydrogenase, glutamate dehydrogenase, aspartate aminotransferase, alanine aminotransferase, pyruvate kinase, creatine kinase, myokinase, alkaline phosphatase and leucine aminopeptidase. In addition plasmaproteins, urea and inorganic phosphorus were measured. In rats blood, was obtained from the following sites: retroorbital venous plexus, jugular vein, heart and ventral aorta. In mice blood was sampled from the jugular vein and the ventral aorta. Shifts of water from the interstitial to the intravascular space due to hypovolemia occuring during the experimental procedure were followed up by measuring the hematocrit and the distribution of radioiodide labelled albumin.

\footnotetext{
1) Gefördert aus Mitteln des Sonderforschungsbereiches 146

2) Teilweise vorgetragen auf der Tagung „Biochemische Analytik 74“
} 
In rats the activities of lactate dehydrogenase, malate dehydrogenase, aspartate aminotransferase, pyruvate kinase, creatine kinase and myokinase found in blood serum obtained from the retroorbital venous plexus and the ventral aorta were too high compared to the other sampling sites. Activities of alkaline phosphatase and alanine aminotransferase were slightly elevated when blood was sampled from the punctured retroorbital venous plexus. Small differences in plasmaproteins and hematocrit values were found to be due to acute shifts of water within the extracellular space. In mice the activities of lactate dehydrogenase, malate dehydrogenase, aspartate aminotransferase and myokinase were found to be too high in blood serum obtained from the ventral aorta.

Efflux of enzymes from damaged cells and the interstitial space is likely to be the main source of etror. Slight hemolysis and blood clotting might have caused erroneous results too, but only to a minor extent. The most reliable method for blood sampling in rat and mouse is the cannulation of the jugular vein. The heart puncture can be recommended too. Attention should be paid, however, to the possibility of aspirating disnupted muscle cells through the inserted needle.

\section{Einleitung und Fragestellung}

"It is irrational to improve methodology as long as uncontrolled sampling conditions may cause changes that far exceed the analytical variability. " Dieser Satz von Pedersen (1) aus dem Jahre 1972 charakterisiert eine Problematik, die in der Klinischen Chemie zwar schon seit der Jahrhundertwende bekannt ist, der aber erst in den letzten Jahren im Zuge der Bemühungen um Qualitätskontrolle und Standardisierung Aufmerksamkeit geschenkt wurde. Für die praktische Medizin haben eingehende Untersuchungen eine Vielzahl von möglichen Fehlerquellen bei der Blutentnahme und der der eigentlichen Analytik vorangehenden Aufarbeitung charakterisieren und ihre Bedeutung für die Gesamt-Präzision der Methode sowie die Aussagekraft eines Parameters für diagnostische Zwecke aufzeigen können (siehe hierzu l.c. $(2,3))$.

Angesichts dieser Aktivitäten ist es erstaunlich, daß die Problematik der Probengewinnung, speziell der Entnahme und Verarbeitung von Blut, ganz offensichtlich wenig Beachtung in der tierexperimentellen Medizin gefunden hat. Bei Durchsicht der einschlägigen Literatur fällt vielmehr auf, daß besonders bei kleinen Laboratoriumstieren wie Ratte und Maus die Vielfalt der angewandten Blutentnahmetechniken kaum noch übertroffen werden kann. Besonders häufig werden Punktionen des retroorbitalen Venenplexus, des Herzens, der Aorta, der V. cava oder der Schwanzgefäße durchgeführt, wobei allerdings vermutet werden muß, daß die Bezeichnung „Punktion“ auch für „Incision“ benutzt wird, da z. B. die Punktion der Aorta bei der Maus nicht unbedingt $\mathrm{zu}$ den einfachen, fur die Routine geeigneten Eingriffen gehört. Als häufig benutzte Techniken werden weiter das Dekapitieren und das Abschneiden der Schwanzspitze gefunden; nicht selten findet man lediglich den Satz: „Die Tiere wurden entblutet.“ Andere Untersucher messen der Blutentnahmetechnik offenbar überhaupt keine Bedeutung bei; sie erwähnen allenfalls, daß Blut, Serum oder Plasma als Untersuchungsmaterial diente. In der Regel wird lediglich bei Untersuchungen, in denen Corticosteroide oder Catecholamine bestimmt werden, besonderer Wert auf eine schnell durchführbare und gut reproduzierbare Blutentnahmetechnik gelegt. Im Rahmen von Untersuchungen über die möglichen Ursachen der biologischen Varianz biochemischer und klinisch-chemischer Parameter im Blutserum verschiedener Rattenstämme wurde uns bewußt, welche entscheidende Bedeutung die Blutentnahmetechnik auf sogenannte Normalwerte und ihre Streuung haben kann.

Es war das Ziel der hier vorgelegten Untersuchungen zu prüfen, ob bei Ratte und Maus die Technik der Blutentnahme Einfluß auf Enzymaktivitäten im Serum hat.

\section{Methodik}

Die Untersuchungen wurden mit Ratten des Inzucht-Stammes LEWIS/ZTM, Geschlecht männlich, Gewicht 180-220 g, und Mäusen des Stammes NMRI/Han, Geschlecht männlich, Gewicht $25-35 \mathrm{~g}$, durchgeführt. Die Tiere wurden in Gruppen von etwa 5 (Ratten) und etwa 10 (Mäuse) in Makrolon-Käfigen III bzw. II bei Einstreu mit Weichholzgranulat, Trinkwasserversorgung über Fläschen und Fütterung mit Altromin R 1320 gehalten; Raumtemperatur $22 \pm 2{ }^{\circ} \mathrm{C}$, relative Luftfeuchtigkeit $50 \pm 5 \%$, Licht von 07.00 bis $19.00 \mathrm{Uhr}$.

In Pentobarbital-Narkose $(40 \mathrm{mg} / \mathrm{kg}$ intraperitoneal) wurde Ratten wie Mäusen ein PVC-Katheter in die rechte V. jugularis eingeführt. Bei Ratten erfolgten die Entnahmen von jeweils 500-800 $\mu 1$ Blut mit vier Techniken nach folgendem Zeitschema:

$t=0$ min Punktion des retroorbitalen Venenplexus mit nicht heparinisierten Kapillaren. Auffangen des Blutes direkt in Zentrifugengefäßen; Umlagerung des Tieres.

$t=1$ min Simultane Entnahme über den Venenkatheter und durch transthorakale Herzpunktion in Plastik-Spritzen; anschließend Laparotomie.

$\mathrm{t}=2 \mathrm{~min}$ Schnittincision der abdominalen Aorta, direktes Auffangen des Blutes in Zentrifugengefäßen.

Den Mäusen wurde innerhalb von maximal 60 s je $300 \mu l$ Blut über den Venenkatheter und durch Incision der abdominalen Aorta entnommen.

Die Blutproben wurden innerhalb weniger Sekunden nach der Entnahme in Polypropylengefäßen für $2 \mathrm{~min}$ mit $12000 \mathrm{~g}$ bei Raumtemperatur zentrifugiert. Das überstehende Nativplasma wurde separiert, nach erfolgter Nachgerinnung wurde das Fibringerinnsel ausgedrückt und entfernt. Die Seren, sie waren frei von sichtbarer Hämolyse, gelangten sofort zur Untersuchung. 
Meßmethoden

Die Aktivitäten von 11 Zellenzymen im Serum wurden mit folgenden Testansätzen (Endkonzentrationen) bestimmt:

Sorbitdehydrogenase, EC 1.1.1.14 (modifiziert nach l.c. (4)): Triäthanolamin-Puffer, pH 7,4, $110 \mathrm{mmol} / \mathrm{l}$; NADH $0,20 \mathrm{mmol} / \mathrm{l}$; Fructose $170 \mathrm{mmol} / \mathrm{l}$.

Lactatdehydrogenase, EC 1.1.1.27 (modifiziert nach 1.c. (5)): Triäthanolamin-Puffer, $\mathrm{pH} 7,5,50 \mathrm{mmol} / \mathrm{l}$; NADH 0,35 $\mathrm{mmol} / \mathrm{l}$; Pyruvat $0,55 \mathrm{mmol} / \mathrm{l}$.

Malatdehydrogenase, EC 1.1.1.37 (modifiziert nach 1.c. (6)): Triäthanolamin-Puffer, $\mathrm{pH} 7,5,50 \mathrm{mmol} / \mathrm{l}$; NADH 0,35 $\mathrm{mmol} / 1$; Oxalacetat $2,0 \mathrm{mmol} / \mathrm{l}$.

Glutamatdehydrogenase, EC 1.4.1.3 (modifiziert nach 1.c. (7)): Triäthanolamin-Puffer, pH 7,5, $35 \mathrm{mmol} / 1$; EDTA 3,5 mmol/1; Ammoniumacetat $100 \mathrm{mmol} / \mathrm{l} ; \mathrm{NADH} 0,28 \mathrm{mmol} / \mathrm{l} ;$ ADP $1,4 \mathrm{mmol} / \mathrm{l} ; 2-O x o g l u t a r a t ~ 6,7 \mathrm{mmol} / \mathrm{l}$.

Aspartataminotransferase, EC 2.6.1.1 (modifiziert nach 1.c. (8)): Phosphat-Puffer, pH 7,4, $80 \mathrm{mmol} / 1 ; L$-Aspartat $200 \mathrm{mmol} / 1$; NADH $0,18 \mathrm{mmol} / 1$; 2-Oxoglutarat $12 \mathrm{mmol} / 1$; Lactatdehydrogenase $1,2 \mathrm{U} / \mathrm{ml}$; Malatdehydrogenase $0,6 \mathrm{U} / \mathrm{ml}$.

Alaninaminotransferase, EC 2.6.1.2 (modifiziert nach 1.c. (9)): Phosphat-Puffer, pH 7,4, $80 \mathrm{mmol} / \mathrm{l} ; L$-Alanin $800 \mathrm{mmol} / \mathrm{l}$; NADH $0,18 \mathrm{mmol} / \mathrm{l} ; 2-O x$ oglutarat $18 \mathrm{mmol} / \mathrm{l}$; Lactatdehydrogenase $1,2 \mathrm{U} / \mathrm{ml}$.

Pyruvatkinase, EC 2.7.1.40 (modifiziert nach l.c. (10)): Triäthanolamin-Puffer, $\mathrm{pH} 7,5,110 \mathrm{mmol} / \mathrm{l} ; \mathrm{K}^{+} 85 \mathrm{mmol} / \mathrm{l}$; $\mathrm{Mg}^{++} 15 \mathrm{mmol} / \mathrm{l}$; EDTA $1,0 \mathrm{mmol} / \mathrm{l}$; NADH $0,19 \mathrm{mmol} / \mathrm{l}$; Phosphocnolpyruvat $0,17 \mathrm{mmol} / \mathrm{l}$; Dithioery thrit $0,2 \mathrm{mmol} / \mathrm{l}$; ADP $1,3 \mathrm{mmol} / \mathrm{l}$; Lactatdehydrogenase $5 \mathrm{U} / \mathrm{ml}$.

Creatinkinase, EC 2.7.3.2 (modifiziert nach 1.c. (11)): Triäthanolamin-Puffer, pH 7,0, $90 \mathrm{mmol} / 1$; Glucose $20 \mathrm{mmol} / \mathrm{l}$; $\mathrm{Mg}^{++} 10 \mathrm{mmol} / \mathrm{l}$; ADP $1,0 \mathrm{mmol} / \mathrm{l}$; AMP $10 \mathrm{mmol} / \mathrm{l}$; NADP 0,7 $\mathrm{mmol} / \mathrm{l}$; Creatinphosphat $35 \mathrm{mmol} / \mathrm{l}$; Dithioerythrit 1,2 $\mathrm{mmol} / \mathrm{l}$; Hexokinase $1 \mathrm{U} / \mathrm{ml}$; Glucose-6-phosphatdehydrogenase $1 \mathrm{U} / \mathrm{ml}$.

Myokinase, EC 2.7.4.3 (modifiziert nach 1.c. (12)): Triäthanolamin-Puffer, pH 7,6, $70 \mathrm{mmol} / \mathrm{l} ; \mathrm{Mg}^{++} 0,7 \mathrm{mmol} / \mathrm{l}$; $\mathrm{K}^{+} 120 \mathrm{mmol} / \mathrm{l}$; NADH 0,54 mmol/1; Phosphoenolpyruvat $0,4 \mathrm{mmol} / \mathrm{l}$; AMP $1,3 \mathrm{mmol} / 1$; ATP $1,1 \mathrm{mmol} / 1$; Lactatdehydrogenase $22 \mathrm{U} / \mathrm{ml}$; Pyruvatkinase $8 \mathrm{U} / \mathrm{ml}$.
Alkalische Phosphatase, EC 3.1.3.1 (13):

Diäthanolamin-Puffer, pH 9,8, 1,0 mol/1; p-Nitrophenylphosphat $10 \mathrm{mmol} / 1 ; \mathrm{Mg}^{++} 0,5 \mathrm{mmol} / 1$.

Leucinaminopeptidase, EC 3.4.11.1 (modifiziert nach 1.c. (14)): Phosphat-Puffer, pH 7,2, $90 \mathrm{mmol} / \mathrm{l}, L$-Leucin-p-nitroanilid $0,8 \mathrm{mmol} / \mathrm{l}$.

Die Bestimmungen erfolgten im optischen Test bei $366 \mathrm{~nm}$, bzw. für die Alkalische Phosphatase und Leucinaminopeptidase kolorimetrisch bei $405 \mathrm{~nm}$; es wurde mit MikroliterMethoden (6) gearbeitet. Alle Messungen erfolgten bei $25^{\circ} \mathrm{C}$ in teilmechanisierten Meßplätzen; die Aktivitäten wurden als $\mathrm{U} / 1$ berechnet.

Der Protein-Gehalt der Scrum-Proben wurde mit Hilfe der Biuret-Reaktion bestimmt (15). Harnstoff und anorganisches Phosphat wurden nach Standardmethoden (16 bzw. 17) unter Verwendung kommerzieller Testpackungen (BOEHRINGER bzw. MERCK) gemessen. Die Bcstimmungen der drei letztgenannten Parameter erfolgten mit einem cinkanaligen diskontinuierlichen Analysenautomaten (Braun SysteMatik). Zur internen Qualitätskontrolle wurden käufliche Kontrollseren (Precinorm E, Precipath E, Precinorm S, sämtlich BOEHRINGER, Mannheim) verwendet. Bci den Ratten wurde der Hämatokrit der Blutproben mit einer Standard-Mikromethode bestimmt; es wurde für $15 \mathrm{~min}$ mit $20000 \mathrm{~g}$ zentrifugiert. Die Untersuchung relativer Veränderungen der Größe des Plasmaraumes erfolgte über die Messung der Verteilung und Elimination intravenös injizierten ${ }^{125} \mathrm{~J}$-markierten Humanalbumins (AMERSHAM-BUCHLER, Braunschweig).

\section{Ergebnisse und Diskussion}

\section{Untersuchungen an Ratten}

In der Tabelle 1 sind die Ergebnisse der Untersuchungen an Ratten zusammengestellt. Für die Enzyme Sorbitdehydrogenase, Glutamatdehydrogenase und Leucinaminopeptidase wurden nahezu identische Aktivitäten bei den vier Entnahmetechniken gefunden. Lediglich bei der Aortenincision lagen die Mittelwerte von Sorbitdehydrogenase und Leucinaminopeptidase niedriger als bei den anderen drei Techniken, die Unterschiede

Tab. 1. Enzymaktivitäten (U/l) und Konzentrationen von Protein (g/l), Harnstoff (mmol/l) und anorganischem Phosphat (mmol/l) im Blutserum sowie der Hämatokrit der Ratte bei Entnahme des Blutes durch vier verschiedene Techniken. $n=12$

\begin{tabular}{|c|c|c|c|c|c|c|c|c|}
\hline & \multicolumn{2}{|c|}{ Retroorbitalpunktion } & \multicolumn{2}{|c|}{ Herzpunktion } & \multicolumn{2}{|c|}{ Venenkatheter } & \multicolumn{2}{|c|}{ Aortenincision } \\
\hline & $\overline{\mathbf{x}}$ & $s_{\overline{\mathbf{x}}}$ & $\bar{x}$ & $s_{\bar{x}}$ & $\overline{\mathbf{x}}$ & $s_{\bar{x}}$ & $\overline{\mathbf{x}}$ & $s_{\overline{\mathbf{x}}}$ \\
\hline $\begin{array}{l}\text { Sorbitdehydrogenase } \\
\text { Lactatdehydrogenase } \\
\text { Malatdehydrogenase } \\
\text { Glutamatdehydrogenase } \\
\text { Aspartataminotransferase } \\
\text { Alaninaminotransferase } \\
\text { Pyruvatkinase } \\
\text { Creatinkinase } \\
\text { Myokinase } \\
\text { Alkalische Phosphatase } \\
\text { Leucinaminopeptidase }\end{array}$ & $\begin{array}{c}6,1 \\
58,8 \\
130 \\
8,8 \\
48,9 \\
54,0 \\
73,5 \\
142 \\
81,6 \\
272 \\
13,3\end{array}$ & $\begin{array}{r}0,5 \\
8,5 \\
25,5 \\
0,8 \\
5,9 \\
4,3 \\
5,8 \\
32,5 \\
16,9 \\
13,9 \\
0,8\end{array}$ & $\begin{array}{r}6,1 \\
45,6 \\
70,3 \\
7,8 \\
37,9 \\
46,7 \\
64,5 \\
104 \\
52,0 \\
235 \\
13,0\end{array}$ & $\begin{array}{r}0,7 \\
5,0 \\
12,1 \\
0,8 \\
1,6 \\
2,8 \\
6,0 \\
13,0 \\
5,5 \\
12,8 \\
0,5\end{array}$ & $\begin{array}{r}6,2 \\
44,4 \\
62,7 \\
8,4 \\
39,5 \\
46,9 \\
59,1 \\
75,1 \\
47,3 \\
243 \\
13,2\end{array}$ & $\begin{array}{r}0,5 \\
3,3 \\
6,3 \\
0,5 \\
1,8 \\
2,6 \\
3,1 \\
5,5 \\
3,0 \\
12,8 \\
0,5\end{array}$ & $\begin{array}{c}5,3 \\
104 \\
134 \\
8,7 \\
46,2 \\
47,6 \\
98,2 \\
239 \\
199 \\
223 \\
12,0\end{array}$ & $\begin{array}{r}0,5 \\
20,6 \\
24,9 \\
0,8 \\
3,1 \\
3,3 \\
13,5 \\
42,1 \\
45,2 \\
11,8 \\
0,6\end{array}$ \\
\hline $\begin{array}{l}\text { Protein } \\
\text { Harnstoff } \\
\text { Phosphat, anorg. }\end{array}$ & $\begin{array}{r}61,9 \\
7,9 \\
2,5\end{array}$ & $\begin{array}{l}0,8 \\
0,1 \\
0,07\end{array}$ & $\begin{array}{r}59,1 \\
7,8 \\
2,3\end{array}$ & $\begin{array}{l}0,7 \\
0,1 \\
0,04\end{array}$ & $\begin{array}{r}60,9 \\
7,9 \\
2,4\end{array}$ & $\begin{array}{l}0,6 \\
0,1 \\
0,07\end{array}$ & $\begin{array}{r}51,5 \\
8,2 \\
2,4\end{array}$ & $\begin{array}{l}1,1 \\
0,2 \\
0,06\end{array}$ \\
\hline Hämatokrit & 46,4 & 0,6 & 44,3 & 0,6 & 45,2 & 0,6 & 37,7 & 1,4 \\
\hline
\end{tabular}


konnten jedoch statistisch nicht gesichert werden. Für Aspartataminotransferase, Alaninaminotransferase und Alkalische Phosphatase wurden bei Retroorbitalpunktion im Mittel um $13 \%$ bis $21 \%$ höhere Werte gefunden als bei Entnahme des Blutes über den Venenkatheter (für Aspartataminotransferase und Alkalische Phosphatase $2 \mathrm{P}<0,05$, für Alaninaminotransferase $2 \mathrm{P}<0,01$ ). Bei der Aspartataminotransferase war auch die Aktivität im Serum aus Blut, das durch Aortenincision gewonnen war, im Mittel um 17\% höher als bei Entnahme aus dem Venenkatheter $(2 \mathrm{P}<0,05)$.

Besonders auffällige Unterschiede wurden bei der Lactatdehydrogenase, der Malatdehydrogenase, der Pyruvatkinase, der Creatinkinase und der Myokinase beobachtet. Bei diesen fünf Enzymen lagen die Aktivitäten im Blutserum nach Aortenincision im Mittel zwischen 67\% (Pyruvatkinase) und 324\% (Myokinase) über den zugehörigen Aktivitäten im Serum bei Blutentnahme aus dem Venenkatheter. Die Unterschiede waren in jedem Falle statistisch signifikant. Auch nach der Retroorbitalpunktion wurden für diese fünf Enzyme signifikant höhere Serum-Aktivitäten gefunden als nach der Blutentnahme aus der kanulierten V. jugularis. Die mittleren relativen Erhöhungen lagen hier zwischen 24\% (Pyruvatkinase) und 100\% (Creatinkinase). Bei der Lactatdehydrogenase, der Malatdehydrogenase, der Pyruvatkinase und der Myokinase wurden zwischen den Techniken Venen-Katheterisierung und Herzpunktion keine signifikanten Unterschiede gefunden. Für die Creatinkinase waren nach Herzpunktion die SerumAktivitäten um im Mittel $43 \%$ höher als nach der Blutentnahme aus der kanulierten Vene $(2 \mathrm{P}<0,05)$. Besonders hervorzuheben ist, daß bei allen Enzymen, für die Unterschiede zwischen den einzelnen Techniken beobachtet wurden, die Varianzen der Meßwerte bei Entnahme des Blutes über den Venenkatheter niedriger als bei jeder anderen Technik waren.

Die Konzentrationen von Harnstoff und anorganischem Phosphat waren bei allen vier Entnahme-Techniken nahezu identisch. Sowohl die Proteinkonzentrationen als auch die Hämatokritwerte wurden nach Aortenincision um im Mittel 15\% niedriger gefunden als nach Entnahme des Blutes über den Venenkatheter (2P $<0,01)$.

Die relativen Unterschiede gegenüber den Meßwerten, die im Serum bzw. Blut nach Entnahme aus der V. jugularis gefunden wurden $(=100 \%)$, sind in der Abbildung 1 dargestellt; sie wurden für jedes Tier getrennt berechnet und dann gemittelt. Aus dieser Abbildung geht auch hervor, welche der Unterschiede im gepaarten t-Test statistisch gesichert werden konnten. Nicht mit aufgenommen wurden diejenigen Parameter, bei denen in keinem Falle signifikante Unterschiede beobachtet worden waren.

Bei der Beurteilung der beobachteten Unterschiede war zu überlegen, ob die Meßwerte evtl. durch die Besonder-

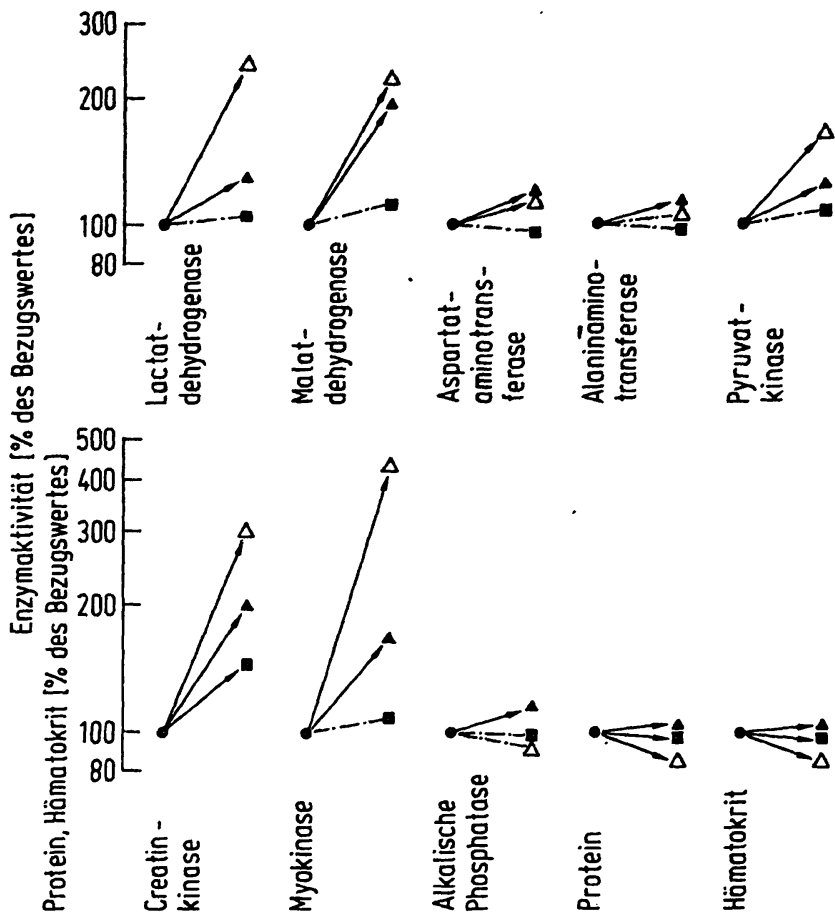

Abb. 1. Relative Unterschiede von Enzymaktivitäten und Proteinkonzentrationen im Blutserum sowie von Hämatokritwerten der Ratte bei Entnahme des Blutes mit vier verschiedenen Techniken.

- Venenkatheter - Herzpunktion

$\Delta$ Retroorbitalpunktion $\triangle$ Aortenincision

$\rightarrow: 2 \mathrm{P}<0,05 \cdot-\cdot-\cdot:$ nicht signifikant

heiten der Versuchsanordnung verfälscht worden waren, da die vier Blutentnahmen aus statistischen Gründen in festgelegter Reihenfolge am gleichen Tier erfolgten. Besonders die Abnahme der Proteinkonzentrationen und der Hämatokritwerte bei der letzten Entnahme legten die Vermutung nahe, daß es im Verlaufe des Versuches $\mathrm{zu}$ akuten Flüssigkeitsverschiebungen zwischen dem intravasalen und dem interstitiellen Raum gekommen war. Die Ratten hatten schon vor der letzten Blutentnahme innerhalb von 1,5-2 min 2-2,5 ml Blut verloren, was bei einem Blutvolumen von $15-20 \mathrm{ml}$ zu einem hypovolämischen Schock führen muß. Zur Klärung dieser Fragestellung wurde die Verteilung bzw. Elimination von intravenös injiziertem ${ }^{125} \mathrm{~J}$-markiertem Albumin untersucht.

Die Abbildung 2 faßt die Ergebnisse von jeweils 9 Versuchen zusammen. Bei der Kontrollgruppe wurden in kurzen Zeitabständen je 200-300 $\mu$ l Blut über einen Venenkatheter entnommen; im Plasma wurde die Radioaktivität gemessen. Daraus resultiert die durchgezogene Kurve, die für Ratten die normale Eliminations-Charakteristik von Makromolekülen aus dem Intravasalraum repräsentiert. Der Versuchsgruppe wurden gleiche Mengen markierten Albumins injiziert. Nach der ersten Blutentnahme zur Ermittlung des Ausgangswertes $(=100 \%)$ erfolgten die weiteren Entnahmen wie im Hauptversuch, sowohl die Volumina als auch die Zeitdifferenzen betreffend. Aus der Abweichung dieser Werte, sie sind als 


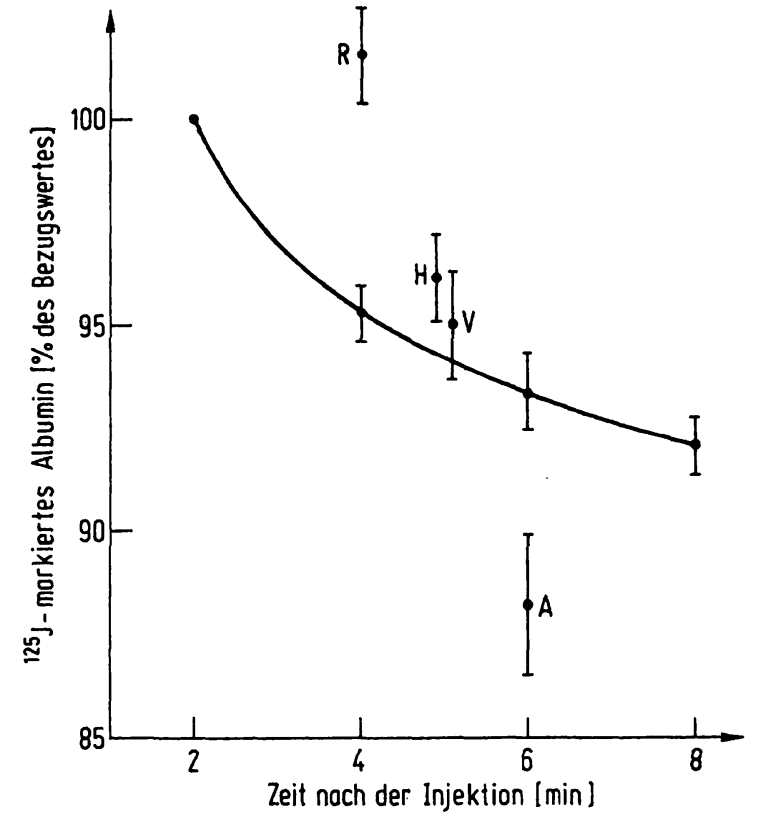

Abb. 2. Einfluß der Blutentnahmetechnik auf die Größe des Verteilungsraumes von intravenös injiziertem ${ }^{125} \mathrm{~J}$-markiertem Albumin. Durchgezogene Linie: Kontrollgruppe; R: Retroorbitalpunktion; H: Herzpunktion; V: Venenkatheter; A: Aortenincision. Mittelwerte und Streuung $\left(s_{\bar{x}}\right)$ aus jeweils 9 Versuchen. Weitere Erklärung im Text.

Punkte mit Streuung in die Abbildung aufgenommen, vom Verlauf der Kontrollkurve kann errechnet werden, daß bei der Retroorbitalpunktion der Verteilungsraum für Albumin um etwa $6 \%$ zu niedrig $(2 P<0,001)$, bei der abschließenden Aortenincision um etwa $6 \%$ zu hoch $(2 \mathrm{P}<0,05)$ erfaßt wurde. Im Falle der Retroorbitalpunktion ist dieser zu einer Erhöhung von Enzymaktivitäten und Proteinkonzentrationen führende Effekt durch einen mit der Stauung beider Venae jugulares verbundenen erhöhten hydrostatischen Druck erklärt. Bei der Aortenincision dürfte es sich um einen durch den akuten Blutverlust bedingten Wassereinstrom aus dem Interstitium handeln. In beiden Fällen. können diese Effekte zwar die Unterschiede in den Proteinkonzentrationen und Hämatokritwerten hinlänglich erklären, nicht aber die Unterschiede für die Enzymaktivitäten.

Es ist naheliegend, bei der Frage nach den Ursachen für die beschriebenen Effekte in erster Linie an störende Einflüsse durch eine leichte Hämolyse oder frühzeitig einsetzende Blutgerinnung zu denken. Das Ausmaß der Aktivitätsunterschiede wie auch die Enzymmuster lassen einen überwiegenden Hämolyse-Effekt absolut unwahrscheinlich erscheinen, zumal in keinem Falle eine sichtbare Hämolyse beobachtet wurde. Nach eigenen Erfahrungen wird bei der Ratte eine Hämolyse sicher dann erkannt, wenn die Konzentration des freien Hämoglobins im Serum oberhalb 0,3 g/l liegt. Eine zu dieser Konzentration führenden Lysis von Erythrocyten in vitro würde zu folgenden Aktivitätsanstiegen führen: Lactatdehydrogenase $36 \mathrm{U} / 1$, Malatdehydrogenase $16 \mathrm{U} / 1$,
Aspartataminotransferase $0,9 \mathrm{U} / 1$, Alaninaminotransferase 0,2 U/1, Myokinase $6 \mathrm{U} / 1$, Creatinkinase kein Anstieg (Diese Berechnung basiert auf unveröffentlichten Daten von Friedel \& Mattenheimer).

Die tatsächlich gemessenen Unterschiede waren in jedem Fall weit größer. Auch zeitliche Unterschiede beim Einsetzen der Blutgerinnung, die gerade bei der Ratte einen massiven Enzymaustritt aus Blutplättchen zur Folge hat (18-20), können die beschriebenen Effekte nicht annähernd erklären; die Enzymmuster in Blutplättchen (19) unterscheiden sich wesentlich vom Muster der beobachteten Aktivitätsunterschiede.

Es ist sehr viel wahrscheinlicher, daß sowohl bei der Retroorbitalpunktion als auch bei der Incision der Aorta dem Blut intrazelluläre wie auch interstitielle Flüssigkeit beigemischt wird, was zu einer Verfälschung der Höhe von Enzymaktivitäten im Serum führen muß. Für die Blutentnahme-Technik des Dekapitierens wurde von Meltzer \& Guschwan (21) auf diese Fehlermöglichkeit hingewiesen. Auch die bei Herzpunktion im Vergleich zur Entnahme über den Venenkatheter deutlich erhöht gefundenen Creatinkinase-Aktivitäten dürften unseres Erachtens auf einen durch die Punktion hervorgerufenen Enzymaustritt zurückzuführen sein, da gerade bei dieser Technik die Gefahr besteht, daß wie bei einer Nadelbiopsie Gewebsteile der Interkostalmuskulatur oder des Myocards aspiriert werden können.

\section{Untersuchungen an Mäusen}

Bei der Maus wurden nur zwei Entnahme-Techniken (Venenkatheter und Aortenincision) miteinander verglichen, da ein zu starker Blutverlust vermieden werden sollte. Aus dem gleichen Grunde wurden die Untersuchungen auf die Bestimmung von Enzymaktivitäten beschränkt. Die Ergebnisse sind in der Tabelle 2 zusammengestellt. Die Aktivitäten der Sorbitdehydrogenase, der Glutamatdehydrogenase, der Alaninaminotransferase, der Pyruvatkinase, der Alkalischen Phosphatase und der Leucinaminopeptidase zeigten keine signi-

Tab. 2. Enzymaktivitäten (U/l) im Serum der Maus bei Entnahme des Blutes durch Venenkatheter und durch Aortenincision. $\mathrm{n}=12$

\begin{tabular}{lcccc}
\hline & \multicolumn{2}{c}{ Venenkatheter } & \multicolumn{2}{c}{ Aortenincision } \\
& $\overline{\mathbf{x}}$ & $s_{\overline{\mathbf{x}}}$ & $\overline{\mathbf{x}}$ & \multicolumn{1}{c}{$\overline{\mathbf{x}}^{\mathrm{x}}$} \\
\hline Sorbitdehydrogenasc & 10,8 & 0,9 & 10,3 & 0,8 \\
Lactatdehydrogenase & 163 & 12,5 & 279 & 41,4 \\
Malatdehydrogenase & 242 & 33,9 & 383 & 40,4 \\
Glutamatdehydrogenase & 11,6 & 0,8 & 12,2 & 0,6 \\
Aspartataminotransferase & 28,1 & 2,1 & 39,4 & 3,7 \\
Alaninaminotransferase & 30,3 & 1,2 & 33,7 & 2,4 \\
Pyruvatkinase & 363 & 38,9 & 396 & 42,0 \\
Creatinkinase & 121 & 12,9 & 409 & 54,4 \\
Myokinase & 21,6 & 3,6 & 140 & 33,8 \\
Alkalische Phosphatase & 166 & 15,2 & 158 & 14,5 \\
Leucinaminopeptidase & 8,5 & 0,5 & 8,7 & 0,6 \\
\hline
\end{tabular}


fikanten Unterschiede. Für die Lactatdehydrogenase, die Malatdehydrogenase, die Aspartatdehydrogenase, die Creatinkinase und die Myokinase wurden ähnliche Ergebnisse wie bei cier Ratte ermittelt, wobei auffällt, daß auch bei der Maus die relativen Unterschiede bei den Aktivitäten der Creatinkinase und der Myokinase mit im Mittel $260 \%$ bzw. $560 \%$ am stärksten ausgeprägt waren. Die statistisch gesicherten relativen Unterschiede sind in der Abbildung 3 dargestellt (bei der Lactatdehydrogenase $2 \mathrm{P}<0,05$, sonst $2 \mathrm{P}<0,01$ ).

Wie die Muster der Aktivitätserhöhung, besonders aber die Creatinkinase-Werte zeigen, dürte auch bei der Maus ein Einfluß von Hämolyse oder Gerinnung bzw. ein hierbei auftretender Enzymaustritt aus Blutplättchen (22) zu vernachlässigen sein. Wie schon bei der Ratte sollte die Ursache für die beschriebenen Effekte vornehmlich in einem Enzymaustritt, provoziert hier durch die Schnittincision, zu suchen sein, zumal wenn man berücksichtigt, daß gerade für die betroffenen Enzyme in der Muskulatur (Muscularis der Aorta!) ein beträchtlicher Aktivitätsgradient $\mathrm{zwischen} \mathrm{dem} \mathrm{intrazellulären} \mathrm{und}$ dem extrazellulären Raum besteht.

Die beschriebenen Ergebnisse sind insofern etwas entmutigend, als gerade die für chronische Versuche elegante Entnahmetechnik der Retroorbitalpunktion bei Untersuchungen von Enzymaktivitäten in der Regel nicht geeignet erscheint. Allenfalls für toxikologische Untersuchungen wären die Fehler, mit denen hier beispielsweise bei den Aminotransferasen zu rechnen ist, tolerierbar. Für Studien zur Aufklärung der Ursachen der biologischen Varianz von Enzymaktivitäten im Serum sind hingegen sowohl die Retroorbitalpunktion als auch die Aortenincision als Techniken der Blutentnahme abzulehnen. Es ist anzunehmen, daß die im Vergleich zur klinischen Medizin extrem hohen Varianzen von Enzymaktivitäten im Serum von kleinen Laboratoriums-

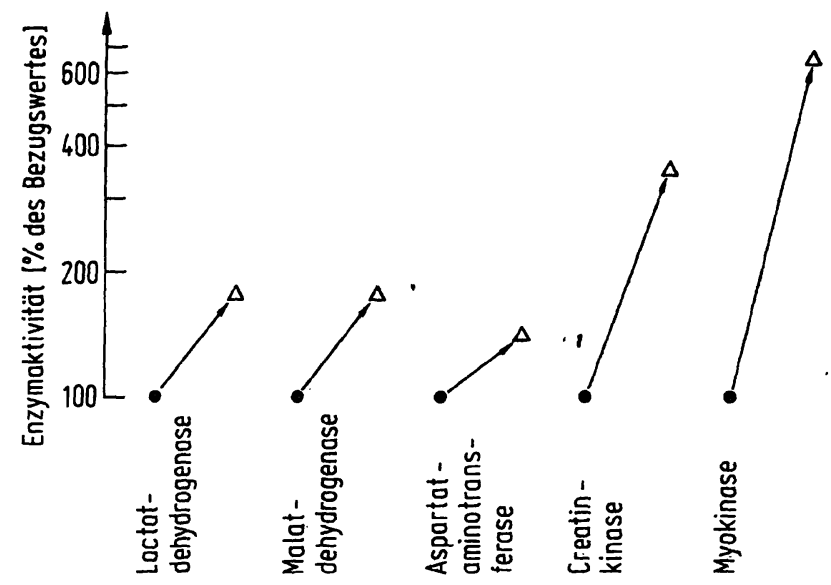

Abb. 3. Relative Unterschiede der Aktivitäten von 5 Zellenzy: men im Serum der Maus bei Entnahme des Blutes über einen Venenkatheter $(\bullet)$ und durch Aortenincision $(\Delta)$. Alle Unterschiede im gepaarten t-Test statistisch signifikant.

tieren (Übersicht bei 1.c. (23)) zumindest teilweise auf methodische Einflüsse zurückzuführen șind.

Für den akuten Versuch ist die Kanulierung der V. jugularis zur Blutentnahme als Methode der Wahl zu bezeichnen, vorausgesetzt, daß der Zeitaufwand durch die Narkose und den relativ einfachen Eingriff akzeptiert werden kann. Mit Einschränkungen kann die Herzpunktion empfohlen werden, wobei allerdings im Falle der Creatinkinase Vorsicht geboten erscheint. Beim chronischen Versuch scheidet der Venenkatheter in aller Regel aus. Vorausgesetzt, daß die Intervalle zwischen den einzelnen Entnahmen größer als eine Woche sind, kann hierfür nur zur Herzpunktion geraten werden. Leider ist dabei aber nach unseren Erfahrungen damit zu rechnen, daß bis zu $10 \%$ der Tiere den Eingriff nicht überleben. Sehr viel optimistischere Angaben von Weis \& Baas (24) konnten von uns nicht bestätigt werden.

\section{Literatur}

1. Pedersen, K. O. (1972), Scand. J. Clin. Lab. Invest. 30 , 191-199.

2. Oette, K. (1973), in Optimierung der Diagnostik (Lang, H., Rick, W. \& Roka, L., Hrs.) S. 103, Springer, Berlin/Heidelberg/New York.

3. Kreutz, F. H. (1973), in l.c. (2), S. 149.

4. Gerlach, U. \& Hiby, W. (1970), in Methoden der enzymatischen Analyse (Bergmeyer, H. U., Hrsg.) S. 527, Verlag Chemie, Weinheim. 5. Richterich, R. (1971), Klinische Chemie, S. 370, Karger,
Basel.

6. Mattenheimer, H. (1966), Mikromethoden für das klinischchemische und biochemische Laboratorium, S. $128, \mathrm{de}^{\circ}$
Gruyter, Berlin.

7. Schmidt, E. (1970), in 1.c. (4), S. 607.
8. Bergmeyer, H. U. \& Bernt, E. (1970), in 1.c. (4), S. 685.

9. Bergmeyer, H. U. \& Bernt, E. (1970), in l.c. (4), S. 715.

10. Gutmann, I. \& Bernt, E. (1970), in 1.c. (4), S. 739. .

11. Forster, G., Bernt, E. \& Bergmeyer, H. U. (1970), in 1.c. (4), S. 750.

12. Bergmeyer, H. U., Gawehn, K. \& Graßl, M. (1970), in 1.c. (4), S. 447.

13. Deutsche Gesellschaft für Klinische Chemie (1972), diese Z. $10,182-192$

14. Nagel, W., Willig, F. \& Schmidt, F. H. (1964), Klin. Wochenschr. 42, 447-449

15. Weichselbaum, T. E. (1946), Amer. J. Clin. Pathol. 10, 40-49

16. Fawcett, J. K. \& Scott, J. E. (1960), J. Clin. Pathol. 13, 156-159 
17. Raabe, S. (1955), Rec. Trav. Chim. Pays-Bas 74, 652-660.

18. Papadopoulos, N. M., Leon, A. S. \& Bloor, C. M. (1967), Proc. Soc. Exp. Biol. Med. 125, 999-1002.

19. Friedel, R. \& Mattenheimer, H. (1970), Clin. Chim. Acta $30,37-46$.

20. Breuer, J. (1974), diese Z. 12, 230.

21. Meltzer, H. \& Guschwan, A. (1971), Amer. Heart J. 82, 138.
22. Friedel, R. \& Mattepheimer, H. (1971), diese Z. 9, 103-106.

23. Bube, P. (1973), Zur Größe der Streuung physiologischer und blutchemischer Parameter in der experimentellen Biologie und Medizin. Inaugural-Dissertation, Tierärztliche Hochschule Hannover.

24. Weis, H. J. \& Baas, E. U. (1971), Z. Ges. Exp. Med. 156, 314-316.
Priv.Doz. Dr. R. Friedel D-3000 Hannover-Kleefeld Karl-Wiechert-Allee 9 
